\title{
New Universality classes for generic higher character Lifshitz points
}

\author{
Marcelo M. Leite* \\ Departamento de Física, Instituto Tecnológico de Aeronáutica, Centro Técnico Aeroespacial, 12228-900, São José dos \\ Campos, SP, Brazil
}

\begin{abstract}
We describe new universality classes associated to generic higher character Lifshitz critical behaviors for systems with arbitrary short range competing interactions. New renormalization-group arguments are proposed for anisotropic and isotropic systems. The usual m-axial Lifshitz universality classes are obtained as a simple limit of this arbitrary competing critical behavior.
\end{abstract}

The universality classes characterizing the critical behavior of competing systems are natural generalizations of those describing ordinary short-ranged critical systems. In magnetic systems [1], the axial next-nearestneighbor Ising (ANNNI) model [2] explains this generalized critical behavior, as antiferromagnetic exchange interactions between second neighbor spins are included along a single axis in a cubic lattice. The uniaxial Lifshitz point arises at the confluence of the disordered, a uniformly ordered and a modulated phase. Second character Lifshitz points generalize the uniaxial one when the competing axes occur in arbitrary space directions. Physical applications of this behavior include magnetic modulated materials $[3,4]$, high- $T_{c}$ superconductors, liquid crystals, among others [5]. The theoretical description of this criticality has been put forth using different field-theoretic tools [6-8] and numerical Monte Carlo simulations for the ANNNI model [9].

The ANNNI model can be generalized by including further ferromagnetic couplings between third neighbors along the competing axis. The phase diagram is characterized by two parameters varying with the temperature, namely, the ratio of exchange couplings between the second and the first neighbors as well as the ratio of exchange interactions between the third and the first neighbors. The projection of the temperature axis in the plane of these parameters results in a region of intersection where the different phases characterizing the system meet, known as a uniaxial Lifshitz point of third character [10]. Including more alternate couplings along the competing direction, the number of parameters in the phase diagram increases. Analogously, the uniaxial Lifshitz point of higher character is defined at the confluence of the distinct phases of the system. The $m_{L}$-fold Lifshitz point of character $L$ arises when alternate couplings are permitted up to the $L$ th neighbors along $m_{L}$ directions and the ratios of the exchange interactions between different neighbors are kept fixed at the associated Lifshitz temperature $[11,12]$.

Lifshitz critical behaviors share at least two issues with a broad class of theories in other physical arenas. In some phenomenological models of quantum gravity, the Planck scale and the speed of light are two observer-independent scales which induce modifications of the usual energymomentum dispersion relations [13]. A nonlinear realization of Lorentz invariance is maintained and can be generalized to include more than two relevant scales [14]. Lorentz invariance can also be preserved in addressing the dark matter problem in cosmology by making use of a scalar field with higher derivative terms, named " $k$ essence" [15]. Alternatively, Planck scale effects may break Lorentz invariance in the ultraviolet regime due to the modification of the dispersion relations. Effective free quantum field theories with modified cubic kinetic terms have just been proposed [16] to test this idea. Thus, a renormalization group analysis with several independent length scales together with new perturbative techniques to solve interacting higher derivative field theories are highly desirable to elucidating new effects in a broader context.

In this letter, we introduce the most general $d$ dimensional competing system resulting in a new static critical behavior by permitting only nearest neighbor interactions along $\left(d-m_{2}-\ldots-m_{L}\right)$ directions, second neighbor competing interactions along $m_{2}$ directions, etc., up to $L$ th neighbor competing interactions along $m_{L}$ directions. The new universality classes for this system generalize the higher character universality classes in a nontrivial way. Since each competing direction defines distinct modulated phases, the generic higher character Lifshitz point arises at the confluence among the disordered, a uniformly ordered and several modulated ordered phases. The universality classes for this arbitrary competing exchange couplings Ising model, named hereafter CECI model, depend on $\left(N, d, m_{2}, \ldots, m_{L}\right)$ where $N$ is the number of components of the order parameter. The

*e-mail:leite@fis.ita.br 
particular isotropic universalities $\left(d=m_{n}\right)$ are obtained independently. We find multiscale scaling relations using a simple scaling hypothesis consistent with homogeneity. Then, we develop a new dimensional regularization procedure to calculate Feynman diagrams and critical exponents for generic higher character Lifshitz points up to two-loop order.

We start with the following bare Lagrangian density:

$$
\begin{aligned}
L= & \frac{1}{2}\left|\nabla_{\left(d-\sum_{n=2}^{L} m_{n}\right)} \phi_{0}\right|^{2}+\sum_{n=2}^{L} \frac{\sigma_{n}}{2}\left|\nabla_{m_{n}}^{n} \phi_{0}\right|^{2} \\
& +\sum_{n=2}^{L} \delta_{0 n} \frac{1}{2}\left|\nabla_{m_{n}} \phi_{0}\right|^{2}+\sum_{n=3}^{L-1} \sum_{n^{\prime}=2}^{n-1} \frac{1}{2} \tau_{n n^{\prime}}\left|\nabla_{m_{n}}^{n^{\prime}} \phi_{0}\right|^{2} \\
& +\frac{1}{2} t_{0} \phi_{0}^{2}+\frac{1}{4 !} \lambda_{0} \phi_{0}^{4} .
\end{aligned}
$$

At the Lifshitz point, the fixed ratios among the exchange couplings explained above translate into this fieldtheoretic version though the conditions $\delta_{0 n}=\tau_{n n^{\prime}}=0$. All even momentum powers up to $2 L$ become relevant in the free propagator, realizing a situation considered by Wilson in the 1970's [17].

The critical space dimension $d_{c}$ above which the classical (mean field) behavior takes over the system is given by $d_{c}=4+\sum_{n=2}^{L} \frac{(n-1)}{n} m_{n}$. The small parameter for a consistent perturbative expansion is $\epsilon_{L}=$ $4+\sum_{n=2}^{L} \frac{(n-1)}{n} m_{n}-d$.

In momentum space, the $m_{n}$ competing axes only possess the term $k_{(n)}^{2 n}$ due to the Lifshitz condition. Thus, we can set $\sigma_{n}=1$ and perform a dimensional redefinition of the competing momenta defining arbitrary competing axes. Hence, if the canonical dimension (in mass units $\Lambda$ ) of the quadratic noncompeting momenta is given by $[q]=\Lambda$, then $\left[k_{(n)}\right]=\Lambda^{\frac{1}{n}}$. Therefore, the volume element in momentum space for arbitrary Feynman integrals has canonical dimension $\Lambda^{d-\sum_{n=2}^{L} \frac{(n-1)}{n} m_{n}}$. Let $\xi_{1}, \xi_{2}, \ldots, \xi_{L}$ be the correlation lengths characterizing arbitrary spatial directions of the system. Close to the Lifshitz critical temperature these quantities are related among each, but can be considered as independent relevant length scales in the problem as follows.

Consider $L$ normalization conditions for the oneparticle irreducible (1PI) vertex parts. The renormalization of the vertices at the Lifshitz temperature is consistent in the infrared limit if the theory is renormalized at nonvanishing external momenta. Let $\kappa_{n}$ be the external momenta scale used to fix the renormalized theory along arbitrary space directions. For instance, for $n=1$ the nonvanishing components of the external momenta are parallel to the noncompeting $\left(d-\sum_{n=2}^{L} m_{n}\right)$-dimensional subspace. For $n>1$, the nonvanishing components of the external momenta occur only along the $m_{n}$-dimensional competing subspace. Let $p_{i(n)}$ be the external momenta associated to a generic vertex part. We can choose several symmetry points $S P_{n}$ along arbitrary directions defined by $p_{i(n)} \cdot p_{j(n)}=\frac{\kappa_{n}^{2}}{4}\left(4 \delta_{i j}-1\right)$. The momentum scale of the two point function is fixed by $p_{(n)}^{2}=\kappa_{n}^{2}=1$. The 1PI renormalized vertex parts at the Lifshitz critical temperature are given in terms of the bare vertices as $[8,18,19](n=1, \ldots, L)$ :

$$
\begin{aligned}
\Gamma_{R(n)}^{(N, L)}\left(p_{i(n)}, Q_{i(n)}, g_{n}, \kappa_{n}\right)= & Z_{\phi(n)}^{\frac{N}{2}} Z_{\phi^{2}(n)}^{L}\left(\Gamma_{(n)}^{(N, L)}\left(p_{i(n)}, Q_{i(n)}, \lambda_{n}, \Lambda_{n}\right)\right. \\
& \left.-\left.\delta_{N, 0} \delta_{L, 2} \Gamma_{(n)}^{(0,2)}\left(Q_{(n)}, Q_{(n)}, \lambda_{n}, \Lambda_{n}\right)\right|_{Q_{(n)}^{2}=\kappa_{n}^{2}}\right)
\end{aligned}
$$

where $g_{n}$ are the renormalized coupling constants, $Z_{\phi(n)}$ and $Z_{\phi^{2}(n)}$ are the field and temperature normalization constants and $\Lambda_{n}$ are the corresponding momentum cutoffs along the noncompeting $(n=1)$ and competing directions $(1<n \leq L)$. Notice that $Q_{i(n)}$ are the insertion external momenta along the different spatial directions for vertex parts including composite $\phi^{2}(x)$ operators.

Our dimensional redefinitions of the momenta components characterizing each type of competing axes implies that the renormalized and bare dimensionful coupling constants can be expressed in terms of dimensionless couplings as $g_{n}=u_{n}\left(\kappa_{n}^{2 n}\right)^{\frac{\epsilon_{L}}{2}}$ and $\lambda_{n}=u_{0 n}\left(\kappa_{n}^{2 n}\right)^{\frac{\epsilon_{L}}{2}}$. Since we are going to analyze the situation for $d<d_{c}$, we can suppress the cutoffs $\Lambda_{n}$ in the argument of the bare vertices recalling, however, that the limit $\Lambda_{n} \rightarrow \infty$ is implicit. The invariance of the bare vertex functions with the renormalizaton scales $\kappa_{n}$ implies the renormalization group (RG) equation

$$
\left(\kappa_{n} \frac{\partial}{\partial \kappa_{n}}+\beta_{n} \frac{\partial}{\partial u_{n}}-\frac{1}{2} N \gamma_{\phi(n)}\left(u_{n}\right)+L \gamma_{\phi^{2}(n)}\left(u_{n}\right)\right) \Gamma_{R(n)}^{(N, L)}=\delta_{N, 0} \delta_{L, 2}\left(\kappa_{n}^{-2 l}\right)^{\frac{\epsilon}{2}} B_{n}\left(u_{n}\right) .
$$


The functions $\beta_{n}, \gamma_{\phi(n)}$ and $\gamma_{\phi^{2}(n)}\left(u_{n}\right)$ are calculated at fixed bare coupling $\lambda_{n}$. In terms of dimensionless quantities, they read

$$
\begin{aligned}
\beta_{n} & =-n \epsilon_{L}\left(\frac{\partial \ln u_{0 n}}{\partial u_{n}}\right)^{-1}, \\
\gamma_{\phi(n)}\left(u_{n}\right) & =\beta_{n} \frac{\partial \ln Z_{\phi(n)}}{\partial u_{n}}, \\
\gamma_{\phi^{2}(n)}\left(u_{n}\right) & =-\beta_{n} \frac{\partial \ln Z_{\phi^{2}(n)}}{\partial u_{n}} .
\end{aligned}
$$

At the fixed points, under flows in the external momenta $p_{i(n)}, Q_{i(n)}$ along arbitrary space directions, the vertex parts $\Gamma_{R(n)}^{(N, L)}\left(\rho p_{i(n)}, \rho Q_{i(n)}, u_{n}^{*}, \kappa_{n}\right)$ have a simple scaling property. As usual, define $\eta_{n}=\gamma_{\phi(n)}^{*}$. The correlation length exponents are given by $\nu_{n}^{-1}=2 n-\gamma_{\phi^{2}(n)}^{*}$. The determination of the scaling relations above and below the Lifshitz critical temperature follows closely that for the second character Lifshitz points $[8,20]$. One finds

$$
\begin{aligned}
\alpha_{n} & =2-n\left(d-\sum_{j=2}^{L} \frac{(j-1)}{j} m_{j}\right) \nu_{n}, \\
\beta_{n} & =\frac{1}{2} \nu_{n}\left(n\left(d-\sum_{i=2}^{L} \frac{(i-1)}{i} m_{i}\right)-2 n+\eta_{n}\right), \\
\gamma_{n} & =\nu_{n}\left(2 n-\eta_{n}\right), \\
\delta_{n} & =\frac{n\left(d-\sum_{i=2}^{L} \frac{(i-1)}{i} m_{i}\right)+2 n-\eta_{n}}{n\left(d-\sum_{i=2}^{L} \frac{(i-1)}{i} m_{i}\right)-2 n+\eta_{n}},
\end{aligned}
$$

which imply the Widom $\gamma_{n}=\beta_{n}\left(\delta_{n}-1\right)$ and Rushbrook $\alpha_{n}+2 \beta_{n}+\gamma_{n}=2$ relations for arbitrary competing or noncompeting subspaces, since $n=1, \ldots, L$.

The isotropic cases are defined by $d=m_{n}$, when all competing interactions not lying on the $m_{n}$ competing axes are switched off. The upper critical dimension is $d_{c}=4 n$ implying that the perturbative parameter is $\epsilon_{4 n}=4 n-d$. There is only one type of correlation length $\xi_{4 n}$. The canonical dimension of the momenta in mass units is redefined to be $[k]=\Lambda^{\frac{1}{n}}$. The volume element of Feynman integrals in momentum space has canonical dimension $\left[d^{d} k\right]=\Lambda^{\frac{d}{n}}$. Following similar steps as done in the anisotropic cases, we obtain the relations:

$$
\begin{aligned}
\gamma_{4 n} & =\nu_{4 n}\left(2 n-\eta_{4 n}\right), \\
\alpha_{4 n} & =2-d \nu_{4 n} \\
\delta_{4 n} & =\frac{d+2 n-\eta_{4 n}}{d-2 n+\eta_{4 n}}, \\
\beta_{4 n} & =\frac{1}{2} \nu_{4 n}\left(d-2 n+\eta_{4 n}\right),
\end{aligned}
$$

which imply the Widom $\gamma_{4 n}=\beta_{4 n}\left(\delta_{4 n}-1\right)$ and Rushbrook $\alpha_{4 n}+2 \beta_{4 n}+\gamma_{4 n}=2$ laws. Note that they trivially reduce to the usual $\phi^{4}$ theory for $n=1$, after the identification $\alpha_{4} \equiv \alpha$, etc.. These relations are the same as those found by Nicoll et al. [21], with the additional scaling relation for $\beta_{n}$ obtained in the present work in a simple manner.

Consider the exponents for the anisotropic cases obtained from the critical theory defined by (2) at nonzero external momenta. At this critical point the bare free critical propagator $(t=0)$ is given by $G_{0}^{-1}=p^{2}+$ $\sum_{n=2}^{L} k_{n}^{2 n}$, where $\vec{p}$ is a wave vector along the $\left(d-m_{2}-\right.$ $\left.\ldots-m_{L}\right)$ noncompeting directions, and $\overrightarrow{k_{n}}$ are the wave vectors along the $m_{n}$ distinct type of competing axes. Let $k_{i(n)}^{\prime}$ be the external momenta along the $m_{n}$ competing axes in the contribution of an arbitrary 1PI vertex part, which at the symmetry points discussed above can be simply written as $k_{(n)}^{\prime}$. If the loop momenta is $k_{(n)}$, we can employ a generalized "orthogonal approximation" [8] to resolving the Feynman integrals up to two-loop level with arbitrary external momenta. Using the approximation $\left(k_{(n)}+k_{(n)}^{\prime}\right)^{2 n} \cong\left(k_{(n)}^{n}+k_{n}^{\prime n}\right)^{2}$, we can redefine the loop momenta as $K_{(n)}=k_{(n)}^{n}$. In each integration over the competing momenta we pick out only the contribution corresponding to a homogeneous function of the momenta. We absorb the geometric angular factor $\left[\left(\Pi_{n=2}^{L} \frac{1}{2 n} S_{m_{n}} \Gamma\left(\frac{m_{n}}{2 n}\right)\right) S_{\left(d-\sum_{n=2}^{L} m_{n}\right)} \Gamma\left(2-\sum_{n=2}^{L} \frac{m_{n}}{2 n}\right)\right]$ in a redefinition of the coupling constant after performing each loop integral. Using (2), we find that the fixed point is indepent of the scaling direction considered and the critical exponents $\nu_{n}$ and $\eta_{n}$ can be calculated diagramatically at two- and three-loop order, respectively. The other exponents are obtained from the scaling laws derived above. They are given by

$$
\begin{aligned}
& \nu_{n}=\frac{\nu_{1}}{n}=\frac{\frac{1}{2}+\frac{(N+2)}{4(N+8)} \epsilon_{L}+\frac{1}{8} \frac{(N+2)\left(N^{2}+23 N+60\right)}{(N+8)^{3}} \epsilon_{L}^{2}}{n} \\
& \eta_{n}=n \eta_{1}=\frac{n}{2} \epsilon_{L}^{2} \frac{N+2}{(N+8)^{2}}\left[1+\epsilon_{L}\left(\frac{6(3 N+14)}{(N+8)^{2}}-\frac{1}{4}\right)\right] \\
& \gamma_{n}=1+\frac{(N+2)}{2(N+8)} \epsilon_{L}+\frac{(N+2)\left(N^{2}+22 N+52\right)}{4(N+8)^{3}} \epsilon_{L}^{2} \\
& \alpha_{n}=\frac{(4-N)}{2(N+8)} \epsilon_{L}-\frac{(N+2)\left(N^{2}+30 N+56\right)}{4(N+8)^{3}} \epsilon_{L}^{2} \\
& \beta_{n}=\frac{1}{2}-\frac{3}{2(N+8)} \epsilon_{L}+\frac{(N+2)(2 N+1)}{2(N+8)^{3}} \epsilon_{L}^{2} \\
& \delta_{n}=3+\epsilon_{L}+\frac{\left(N^{2}+14 N+60\right)}{2(N+8)^{2}} \epsilon_{L}^{2}
\end{aligned}
$$

Notice that the anisotropic exponents $\gamma_{n}, \alpha_{n}, \beta_{n}$ and $\delta_{n}$ easily reproduce those of the second character Lifshitz behavior when $m_{3}=\ldots=m_{L}=0$ with $m_{2}=m \neq 0$, which turn into the Ising-like exponents for $m_{2}=m=0$. The generalized universality classes exposed here and the properties $\nu_{n}=\frac{\nu_{1}}{n}$ and $\eta_{n}=n \eta_{1}$, when $m_{2}=\ldots=$ $m_{L-1}=0$ with $m_{L} \neq 0$, imply that our anisotropic scaling relations are equivalent to those found in Ref. [12], except for the scaling relation of the magnetization exponent which was not obtained there. Strong anisotropic 
scaling [22] is exact at the loop order considered here in the most general anisotropic situation, and might be expected to hold at arbitrary loop levels.

Using the same approximation scheme to perform Feynman integrals as outlined above, the isotropic ex- ponents are even simpler to obtain. The angular factor to be absorbed in the loop integrals is simply the area of the $m_{n}$-dimensional sphere $S_{m_{n}}$. The critical exponents are given by [23]:

$$
\begin{aligned}
& \eta_{4 n}=\frac{1}{2 n} \epsilon_{4 n}^{2} \frac{N+2}{(N+8)^{2}}\left[1+\frac{\epsilon_{4 n}}{n}\left(\frac{6(3 N+14)}{(N+8)^{2}}-\frac{1}{4}\right)\right] \\
& \nu_{4 n}=\frac{1}{2 n}+\frac{(N+2)}{4 n^{2}(N+8)} \epsilon_{4 n}+\frac{1}{8 n^{3}} \frac{(N+2)\left(N^{2}+23 N+60\right)}{(N+8)^{3}} \epsilon_{4 n}^{2} \\
& \gamma_{4 n}=1+\frac{(N+2)}{2 n(N+8)} \epsilon_{4 n}+\frac{(N+2)\left(N^{2}+22 N+52\right)}{4 n^{2}(N+8)^{3}} \epsilon_{4 n}^{2} \\
& \alpha_{4 n}=\frac{(4-N)}{2 n(N+8)} \epsilon_{4 n}-\frac{(N+2)\left(N^{2}+30 N+56\right)}{4 n^{2}(N+8)^{3}} \epsilon_{4 n}^{2} \\
& \beta_{4 n}=\frac{1}{2}-\frac{3}{2 n(N+8)} \epsilon_{4 n}+\frac{(N+2)(2 N+1)}{2 n^{2}(N+8)^{3}} \epsilon_{4 n}^{2} \\
& \delta_{4 n}=3+\frac{\epsilon_{4 n}}{n}+\frac{\left(N^{2}+14 N+60\right)}{2 n^{2}(N+8)^{2}} \epsilon_{4 n}^{2} .
\end{aligned}
$$

The isotropic exponents $\alpha_{4 n}, \beta_{4 n}, \gamma_{4 n}$ and $\delta_{4 n}$ depend explicitly on the number of neighbors coupled through competing exchange interactions $n$ and labels the isotropic universality classes. These critical exponents reduce to those from the Ising-like $\left(\phi^{4}\right)$ universality class for $n=1$. For $n \geq 2$, these exponents can never be obtained from those of the anisotropic cases described above, since the latter do not depend explicitly on $n$. This confirms that the isotropic universality classes are independent from the anisotropic universality classes for Lifshitz points of generic higher character.

In summary, the CECI model proposed here presents the most general sort of second order phase transition for arbitrary types of competition and realizes new universality classes for generic higher character Lifshitz critical behaviors. The scaling relations are obtained through a generalized scaling hypothesis which defines independent renormalization group tranformations in each competing (or noncompeting) subspace. The critical exponents are calculated up to two-loop level using a new analytical dimensional regularization method to compute Feynman integrals with arbitrary even momentum powers in the propagator, which may be fruitful in quantum field theory applications. Universal quantities as amplitude ratios $[24,25]$ can be pursued in this framework. We relegate the details of the results described here for a subsequent publication [26].

It is important to mention that a new anisotropic behavior emerges when $d=m_{2}+\ldots+m_{L}$. The noncompeting directions which tend to favor the existence of the ferromagnetic phase, are absent in this new situation. This particular behavior deserves a complete and careful study for future investigations. The analysis of the most general crossover effects as well as the tricritical behavior of the model are also worthwhile.

I would like to thank S. K. Adhikari and S. R. Salinas for useful comments on the manuscript and financial support from FAPESP, grant number 00/06572-6.

[1] R. M. Hornreich, M. Luban, and S. Shtrikman, Phys. Rev. Lett. 35, 1678 (1975).

[2] W. Selke, Phys. Rep. 170, 213 (1988).

[3] C. C. Becerra, Y. Shapira, N. F. Oliveira Jr., and T. S. Chang, Phys. Rev. Lett. 44, 1692 (1980); C. S. O. Yokoi, M. D. Coutinho-Filho, and S. R. Salinas, Phys. Rev. B 29, 6341 (1984); C. C. Becerra, V. Bindilatti, and N. F. Oliveira Jr., Phys. Rev. B 62, 8965 (2000).

[4] T. Hanawa, K. Shinkawa, M. Ishikawa, K. Miyatani, K. Saito, and K. Kohn, J. Phys. Soc. Jpn. 63, 2706 (1994); H. Weitzel, H. Ehrenberg, C. Heid, and H. Fuess, Phys. Rev. B 62, 12146 (2000).

[5] For a recent review, see W. Selke, in Phase Transitions and Critical Phenomena, edited by C. Domb and J. Lebowitz (Academic Press, London), vol.15 (1998).

[6] C. Mergulhão Jr, and C. E. I. Carneiro, Phys. Rev. B 58, 6047 (1998), ibid. 59, 13954 (1999); H. W. Diehl, and M. Shpot, Phys. Rev. B 62, 12338 (2000); M. Shpot, and H. W. Diehl, Nucl. Phys. B 612(3), 340 (2001).

[7] L. C. de Albuquerque, and M. M. Leite, J. Phys. A: Math Gen. 34, L327 (2001); ibid. 35, 1807 (2002).

[8] M. M. Leite, Phys. Rev. B 67, 104415 (2003). 
[9] M. Pleimling, and M. Henkel, Phys. Rev. Lett. 87, 125702 (2001).

[10] W. Selke, Z. Physik B 27, 81 (1977); W. Selke, Journ. Magn. Magn. Mat. 9, 7 (1978).

[11] W. Selke, Phys. Lett. A 61, 443 (1977).

[12] J. F. Nicoll, T. S. Chang, and H. E. Stanley, Phys. Rev. A 13, 1251 (1976); J. F. Nicoll, G. F. Tuthill, T. S. Chang, and H. E. Stanley, Phys. Lett. A 58, 1 (1976).

[13] G. Amelino-Camelia, Nature 418, 34 (2002); J. Magueijo, and L. Smolin, Phys. Rev. Lett. 88, 190403 (2002).

[14] J. Magueijo, and L. Smolin, Phys.Rev. D 67, 044017 (2002).

[15] C. Armendariz-Picon, V. Mukhanov, and P.J.Steinhardt, Phys. Rev. Lett. 85, 4438 (2000).

[16] R. C. Myers, and M. Pospelov, Phys. Rev. Lett. 90, 211601 (2003).

[17] K. G. Wilson, Rev. Mod. Phys. 47, 773 (1975).

[18] D. J. Amit, in Field Theory, the Renormalization Group and Critical Phenomena (World Scientific, Singapore)(1984).

[19] E. Brézin, J. C. Le Guillou, and J. Zinn-Justin, in Phase Transitions and Critical Phenomena, edited by C. Domb and M. S. Green (Academic Press, London), vol. 6 (1976).

[20] For the anisotropic behavior, one just has to replace $\tau$ in Ref. [8] by $n$ as well as the effective dimension $\left(d-\frac{m}{2}\right)$ in Ref. [8] by $\left(d-\sum_{j=2}^{L} \frac{(j-1)}{j} m_{j}\right)$ and follow the same steps to find the scaling relations given herein. For the generalized isotropic cases one can replace the dimension 8 by $4 n$. The beta function $\beta_{3}$ in Ref. [8] is replaced here by $\beta_{4 n}=-\epsilon_{4 n}\left(\frac{\partial \ln u_{04 n}}{\partial u_{4 n}}\right)^{-1}$.

[21] J. F. Nicoll, G. F. Tuthill, T. S. Chang, and H. E. Stanley, Physica B 86-88, 618 (1977).

[22] M. Henkel, Phys. Rev. Lett. 78, 1940 (1997).

[23] There was a misprint (a factor of $\frac{1}{4}$ ) in the coefficient of the $\epsilon_{L}^{2}$ term in the exponent $\nu_{L 4}$ which was carelessly included in Eq.(208) of Ref. [8]. Except for $\eta_{L 4}$ which has the correct expression therein, the remaining exponents carried this misprint. The correct expressions for these exponents in the particular second character isotropic case $n=2$ are given herein.

[24] M. M. Leite, Phys. Rev. B 61, 14691 (2000).

[25] M. M. Leite, Phys. Rev. B 68, 052408 (2003).

[26] M. M. Leite, in preparation. 